Original Research Paper

\title{
Evaluation of Some Vaccination Programs in Protection of Experimentally Challenged Broiler Chicken against Newcastle Disease Virus
}

\author{
${ }^{1}$ Mousa A. Ayoub, ${ }^{2 \#}$ Wael K. Elfeil, ${ }^{1}$ Diaa El Boraey, ${ }^{3}$ Haitham Hammam and ${ }^{4}$ Mohamed, A. Nossair \\ ${ }^{I}$ Department of Animal Hygiene and Zoonoses, Faculty of Veterinary Medicine, Damanhur University \\ ${ }^{2}$ Department of Avian and Rabbit Medicine, Faculty of Veterinary Medicine, Suez Canal University \\ ${ }^{3}$ Department of Avian and Rabbit Medicine, Faculty of Veterinary Medicine, Aswan University \\ ${ }^{4}$ Department of Animal Hygiene and Zoonoses, Faculty of Veterinary Medicine, Alexandria University
}

Article history

Received: 20-04-2019

Revised: 19-06-2019

Accepted: 20-07-2019

Corresponding Author:

Wael K. Elfeil

Department of Avian and Rabbit Medicine, Faculty of

Veterinary Medicine, Suez

Canal University

Email: wael.elfeil@gmail.com

\begin{abstract}
The current study was carried out to evaluate efficiency of different vaccination programs in protecting broiler chicken against Virulent Viscerotropic Strain of Newcastle disease virus. Different live and inactivated NDV vaccines were used throughout the experiment including; ND-HB1,Elite, LaSota, Inactivated GII and Inactivated GVII. Broiler chicks were divided into 8 groups; 6 groups undergo different vaccination programs against NDV while two groups were kept without vaccination to be the control groups. Challenge was done at day $30^{\text {th }}$ of age via intranasal administration of NDV velogenic GVII (NDV/CK/Egypt/567F/2012). Abs titers were determined at days 1, 7, 14, 21, 28 and 35 days of the experiment. The obtained results of shedding titers of NDV clarified that the lowest shedding titer was recorded in group vaccinated by $\mathrm{HB}+$ double shots of Inactivated GVII + Elite + LaSota and HB + double shots of Inactivated GII + Elite + LaSota, then group vaccinated by $\mathrm{HB}+$ one shot of Inactivated GVII + Elite + LaSota and HB + one shot of Inactivated GII + Elite + LaSota at days 3, 5, 7 and 9 post challenge day at $30^{\text {th }}$, respectively compared to those vaccinated by live vaccines $\mathrm{HB}+$ LaSota only and $\mathrm{HB}+$ Elite + LaSota only. Also, no mortalities (100\% protection rate) were recorded in groups vaccinated with both live and inactivated NDV vaccines compared to low mortality rates recorded in groups vaccinated with live vaccines only. Based on the recorded results, it was concluded that application of ND vaccination programs containing both live and double inactivated vaccines (either GII or GVII) was found to be more effective than those depending on one shot of inactivated vaccine (either GII or GVII) plus live vaccines and more effective than program including live vaccines only.
\end{abstract}

Keywords: ND, Vaccination Programs, Live and Inactivated Vaccines, Shedding

\section{Introduction}

In Egypt, poultry sector is the fastest growing industry, with over 90 billion Egyptian pound investments. $(1 \$=$ approximately 17 L.E.) (Sabry, 2019). It provides the country with the greatest part of its meat production, in addition to job opportunities for approximately 3 million employees (Sabry, 2019). Several pathogenic agents affect the poultry and associated with severe losses in the poultry production in Egypt specially Newcastle virus, Avian influenza, infectious bronchitis, Infectious bursal disease virus and multidrug resistant bacterial pathogens (Diab et al., 2019; Eid et al., 2016;
Eid et al., 2019; Elhady et al., 2018; Sedeik et al., 2018; Sedeik et al., 2019; Sultan et al., 2019). So, presence of diseases like ND that was described in Egypt for the first time in 1948 (Daubney and Mansy, 1948) represents a real threat to Egyptian poultry industry. Newcastle disease (ND) is considered one of the most important infectious diseases all over the world. Nearly it affects birds in the six continents causing major economic losses, prompting Office International des Epizooties to classify it as list A disease (OIE, 2015). The causative agent Newcastle disease virus (NDV) is an enveloped, single stranded, non-segmented RNA of negative sense virus. $\mathrm{NDV}$ is the only member of the genus Avulavirus, 
subfamily Paramyxoviridae that belonged to family Paramyxoviridae in the order Mononegavirales and is designated Avian Paramyxovirus-1 (APMV-1) (Alexander and Senne, 2008). According to virulence, NDV isolates can be classified into three main pathotypes; velogenic (virulent), mesogenic (of moderate virulence) and lentogenic (of low virulence) (Orsi et al., 2009). Virulent $\mathrm{NDV}$ is responsible for the severe clinical symptoms in affected chickens including; diarrhea, nervous and respiratory manifestations, egg problems like low production and production of deformed eggs, hemorrhagic lesions in the trachea, intestine and proventriculus and mortality (Desingu et al., 2017; Guo et al., 2014; Liu et al., 2015). The infected and carrier chickens are the main source of infection to other healthy birds in the flock through shedding the virus in their droppings and respiratory secretions (Nwanta et al., 2008). Vaccination is still the most important tool in controlling ND. There are two main types of vaccines used against NDV; live vaccines and inactivated vaccines. The inactivated type is not practical in application especially in large flocks as they need to be injected and have low immunogeniticity but prevent viral shedding (Mansur-Ud-Din et al., 2007). On the other hand, live low virulent strains are used as live vaccines to give protection against virulent strains depending on the fact of the antigenic similarity between all NDV strains (Miller et al., 2013a). Different studies proved the ability of live NDV vaccines to reduce or prevent clinical disease and mortality, decrease the amount of virus shedding into the environment and increase the amount of virus needed to infect the vaccinated birds (Marangon and Busani, 2007; Miller et al., 2009). In addition to inducing better immunity, live vaccines are more convenient in dealing with large flocks as they can be applied via spraying or drinking water (Dimitrov et al., 2017; Perozo et al., 2012). However, these application methods may result in wide variation in the immune responses of vaccinated birds (Senne et al., 2004). Even with suing live ND vaccines and all its core values, NDV can cause infection in vaccinated susceptible birds and shed virus and increase the epidemiological load and increase risk of infected surround susceptible birds (Kapczynski and King, 2005). Moreover, vaccination with live vaccines may induce disease occurrence and reduce growth rates of vaccinated birds (Swayne and Glisson, 2013). In Egypt, where the ND is endemic, vaccination is applied as a routine prophylactic measure using both live attenuated and inactivated vaccines from day zero of chick age; even with this preventive measure ND outbreak are still known to occur hence we suggest improving the vaccination strategy. So, the current study was carried out to evaluate the efficiency of different vaccination programs in protecting experimentally challenged broiler chicken against VV strain of Newcastle disease virus (NDV) and determination of virus shedding of different groups indicating the extent and period of infectiousness by
qRT-PCR, comparison of Abs titer using Hemagglutination Inhibition (HI) test and feed conversion ratio.

\section{Materials and Methods}

\section{Commercial Broiler Chicks and Experimental Area}

A total of 1200 one-day old Arbor Acres broiler chicks with an average weight of $42 \mathrm{~g}$ obtained from Cairo Poultry Company (CPC) were allotted randomly into eight groups (150 birds /group). Floor rearing under hygienic conditions was applied to birds in previously cleaned and disinfected isolated experimental pens where biosecurity measures were implemented. Birds were fed a commercial fattening feed produced by Cairo Poultry Company (CPC) by average of $3.5 \mathrm{~kg}$ food / bird throughout the experiment time, it can be divided into: $700 \mathrm{~g}$ starter / bird of $23 \%$ protein, $2000 \mathrm{~g}$ grower / bird of $21 \%$ protein and $800 \mathrm{~g} /$ bird finisher of $19 \%$ protein. Water and feed were provided ad-libitum. Maternal immunity was measured for 60 randomly selected 1-day old chicks before being divided into groups. Each group was weighted weekly till the end of the experiment where the final body weights were determined.

ND Virus, Vaccines, Reference Antigens and Antisera Used in the Experiment

\section{Newcastle Disease Challenge Virus}

The vNDV $\left(10^{6} \quad\right.$ EID $\left._{50} / 0.5 \mathrm{ml}\right)$ challenge virus NDV/CK/Egypt/567F/2012 (acc. no. JX647839) belongs to genotype VIId currently circulating in Egypt. All viruses were propagated and titrated in 10-day-old SPF eggs. The virus challenge dose equal 6-Log-10 EID $_{50}$ given $0.5 \mathrm{ml} /$ bird (OIE, 2015).

\section{Vaccines}

Different live and inactivated NDV vaccines were used throughout the experiment as illustrated in Table (1). Live vaccines were administrated through eye distillation while inactivated vaccines were administrated through subcutaneous injection in skin fold of the neck.

\section{NDV Reference Antigens and Antisera}

ND LaSota vicinal strain was propagated in ECE and diluted to $4 \mathrm{HAU}$ to be used as $\mathrm{HA}$ antigen in hemagglutination inhibition (HI) titration of ND antibody, was obtained from ME-VAC Egypt, friendly dedicated. Known positive and negative NDV antisera were provided.

\section{Experiment Design}

Broiler Broiler chicks were divided into 8 groups (150 chicks / group). Groups from 1 to 6 undergo different vaccination programs against NDV as illustrated in Table (2) while groups 7 and 8 were 
unvaccinated control groups. Ab titers were determined at days $1,7,14,21,28$ and 35 of the experiment in 15 randomly selected chicks from each group. At day $28^{\text {th }}$ of age ( 2 days before challenge), 15 birds from each group were randomly selected and moved to controlled isolated rooms where they were examined for presence of Avian Influenza virus (H5 \& H9), Newcastle (ND) virus and Infectious Bronchitis (IB) virus using one step RT-PCR at day zero and the two successive days post isolation to ensure their freedom from these pathogens. Challenge was done at day 30 to groups from 1 to 7 via intranasal administration of NDV $\left(10^{6} \mathrm{EID}_{50}\right)$ velogenic GVII (NDV/CK/Egypt/567F/2012). Finally, shedding percentage of NDV was detected in Oropharyngeal (tracheal) swabs by using qRT-PCR at days 3, 5, 7 and 9 post challenges. Chicken blood were collected from wing vein or by slaughtering and kept in slop position at $37^{\circ} \mathrm{C}$ for one hour then at $4^{\circ} \mathrm{C}$ overnight. Sera then separated by centrifugation at $3000 \mathrm{rpm} / 10$ minutes and stored at $-20^{\circ} \mathrm{C}$ till tested by $\mathrm{HI}$ as previously described (OIE, 2015).

\section{Molecular Identification of Viral Pathogens}

The kits used flowing manufacturer instructions as shown in Tabel 3.

\section{Assessment Parameters}

\section{Shedding Titer}

Virus shedding was determined in Oropharyngeal (tracheal) swabs by using qRT-PCR as previously described
(Miller et al., 2007) and the virus titers were calculated using the Spearman-Kärber method (Kärber, 1931):

$$
\text { Mean of shedding titer }=\frac{\text { SUM of virus shedding }}{\text { Total Number of shedders }}
$$

NB. Virus shedding titer (titer $\pm \mathrm{SD}$ ) as $\mathrm{EID}_{50}$ using qRT-PCR with absolute standard curve and the engine detection threshold 0.7 EID $_{50}$.

\section{- Humoral Immunity}

Expressed by titration of Abs by $\mathrm{HI}$ test at age $1,7,14,21,28$ of age and 5 days post challenge.

- Mortality Rate

$$
\text { Mortality rate }=\frac{\text { Number of dead birds }}{\text { total number of birds in each group }} \times 100
$$

\section{- Feed Conversion Ratio}

$F C R$ for each week interval was estimated:

$$
F C R=\frac{\text { Feed intake }(g) \text { in a given period }}{\text { Body weight gain }(g) \text { in the same period }}
$$

\section{Statistical Analysis}

It was made using $\mathrm{Chi}^{2}$ test to examine the significant differences of the detection rate of antibodies among different groups studied using SAS software version 9.4 (Institute, 2014).

Table 1: Description of NDV vaccines used in different vaccination programs

\begin{tabular}{|c|c|c|c|c|c|}
\hline Age of vaccination & Day 1 & Dav 7 & Day 10 & Dav 18 & Dav30 \\
\hline Group 1 & $\mathrm{HB}$ & ND Elite & & LaSota & Challenge by \\
\hline Group2 & HB & & & LaSota & $\operatorname{NDV}\left(10^{6} \mathrm{EID}_{50}\right)$ \\
\hline Group3 & HB & ND Elite + Inactivated GII & & LaSota & velogenic GVII \\
\hline Group4 & $\mathrm{HB}$ & ND Elite + Inactivated GVII & & LaSota & \\
\hline Group5 & $\mathrm{HB}+$ Inactivated GVII & ND Elite & Inactivated GVII & LaSota & \\
\hline Group6 & HB+ Inactivated GII & ND Elite & Inactivated GII & LaSota & Intranasal \\
\hline Group 7 (control + ve) & No vaccination program & & & & \\
\hline Group 8 (control - ve) & No vaccination program & & & & No challenge \\
\hline
\end{tabular}

\begin{tabular}{llll}
\hline Vaccine used & Company & Dose & Strain used/dose \\
\hline ND-HB1 & ME-VAC (Egypt) & $1000 \mathrm{~d} /$ vail & $10^{6}$ EID $_{50}$ of ND strain HB \\
ND-Elite & ME-VAC (Egypt) & $1000 \mathrm{~d} /$ vail & $10^{6}$ EID 50 of ND strain ND-60 \\
ND LaSota & ME-VAC (Egypt) & $1000 \mathrm{~d} /$ vail & $10^{6}$ EID $_{50}$ of ND strain LaSota \\
Inactivated GII & ME-VAC (Egypt) & $1000 \mathrm{~d} /$ vail $0.3 \mathrm{cc}$ & NDV/Chicken/Egypt/11478AF/2011(ND)108 \\
Inactivated GVII (Dalguban) & KBNP (Korea) & $1000 \mathrm{~d} /$ vail0.5 cc & NDV Genotype VII, KBNP-C4152R2L \\
\hline
\end{tabular}

Table 2: Description of vaccination programs applied to the experimental groups 
Table 3: Ready used kits for Q-RT-PCR

\begin{tabular}{lll}
\hline Gene fragment & Kit name & Company \\
\hline NDV- F gene & ABT NDV-F gene QPCR & Applied Biotechnology, Egypt \\
AIV-H5 & ABT AIV-H5 QPCR & Applied Biotechnology, Egypt \\
AIV-H9 & ABT AIV-H9 QPCR & Applied Biotechnology, Egypt \\
IBV-S1 gene & ABT IBV-S1 QPCR & Applied Biotechnology, Egypt \\
\hline
\end{tabular}

\section{Results}

The The recorded results in showed the Oropharyngeal (tracheal) shedding of challenged NDV from broiler chicken at $3^{\text {rd }}$ day post challenge. $\mathrm{Chi}^{2}$ analysis of the obtained results showed significant difference between the shedding titers of NDV of different experimental groups where the lowest shedding titer was recorded in experimental group that was vaccinated by $\mathrm{HB}+$ Inactivated GVII, Elite, Inactivated GVII and LaSota vaccines at days 1, 7, 10 and 18 of age (G5), respectively followed by the experimental group vaccinated by $\mathrm{HB}+$ Inactivated GII, Elite, Inactivated GII and LaSota at the same age (G6), respectively. On the other hand, the highest shedding titer was recorded in experimental group that was not vaccinated and challenged by NDV (G7) followed by the experimental group that was vaccinated by $\mathrm{HB}$, and LaSota vaccines at days 1 and 18 of age (G2), respectively as shown in Table (5).

As shown in Table (5), it was observed that experimental groups that was vaccinated by $\mathrm{HB}+$ Inactivated GVII, Elite, Inactivated GVII and LaSota vaccines (G5) and $\mathrm{HB}+$ Inactivated GII, Elite, Inactivated GII and LaSota (G6) at days 1, 7, 10 and 18 of age, respectively showed no shedding of the NDV at $5^{\text {th }}$ DPC reflecting efficient vaccination program. On the other hand, the highest shedding titer $(4.27 \pm 0.15)$ was recorded in experimental group that was not vaccinated and challenged by NDV (G7) followed by the experimental group that was vaccinated by $\mathrm{HB}$ and LaSota vaccines $(2.92 \pm 1.21)$ at days 1 and 18 of age (G2), respectively. Moreover, it was recorded that birds of the group that was not vaccinated and challenged by NDV were totally dead as a result of ND infection (G7).

The presented data in Table (5) clarified the Oropharyngeal (tracheal) shedding of challenged NDV from broiler chicken at $7^{\text {th }}$ DPC. As $5^{\text {th }}$ DPC, it was observed that experimental groups that was vaccinated by HB + Inactivated GVII, Elite, Inactivated GVII and LaSota vaccines (G5) and HB + Inactivated GII, Elite, Inactivated GII and LaSota (G6) at days 1, 7, 10 and 18 of age, respectively showed no shedding of the NDV reflecting efficient vaccination program. On the other hand, the highest shedding titer $(1.86 \pm 0.56)$ was recorded in experimental group that was vaccinated by $\mathrm{HB}$ and LaSota vaccines at days 1 and 18 of age (G2), respectively followed by experimental group that was vaccinated by HB, Elite and LaSota (G1) (1.68 \pm 0.53$)$.
At $9^{\text {th }}$ DPC, viral shedding occurred only in the group vaccinated by $\mathrm{HB}$ and LaSota at days of 1 and 18 of age (G2) by shedding birds $(30.7 \%)$ and shedding titer of $(1.25 \pm 0.83)$ and the group vaccinated by $\mathrm{HB}$, Elite and LaSota at days of 1,7 and 18 of age (G1) by only shedding birds $(21.4 \%)$ and shedding titer of $(1.05 \pm 0.41)$ (Table 5).

Mortality rate of broiler chicken during the experiment was tabulated in Tables (4). Concerning mortality rate of broiler chicken after challenge by NDV, the highest mortality rate $(100 \%)$ was observed in nonvaccinated challenged group (G7) where no vaccination programs were applied. On contrary, no mortalities (100\% protection rate) were recorded in groups vaccinated with both live and inactivated NDV vaccines (G3, G4, G5, and G6). In addition, low mortality rates were recorded in groups vaccinated with live vaccines only (G1, G2) by (HB, Elite and LaSota).

The presented data in Fig. (1) Showed that 15 randomly selected chicks from each experimental group (150 chicks) at 14 days old were tested by $\mathrm{HI}$ test for determination of $\mathrm{Ab}$ titers against $\mathrm{VDV}$ vaccine. Statistical analysis clarified significant difference at $(\mathrm{P}<$ 0.05 ) among different groups in $\mathrm{X} \log$. The highest $\mathrm{Ab}$ titers was observed in group vaccinated with $\mathrm{HB}+$ Inactivated GII, Elite, Inactivated GII and LaSota vaccines (G6) (Live and double inactivated GII vaccine) (X $\log 4.06 \pm 0.44 \mathrm{~A}$ ) followed by the group vaccinated with HB + Inactivated GVII, Elite, Inactivated GVII and LaSota (G5) (Live and double shot of inactivated GVII vaccine) ( $\mathrm{X} \log 3.86 \pm 0.85 \mathrm{~B})$. On contrary, positive and negative control groups (non-vaccinated) showed the lowest $\mathrm{Ab}$ titers $(\mathrm{X} \log 1.26 \pm 0.20 \mathrm{~F}$ and $1.33 \pm 0.20 \mathrm{~F}$, respectively) (G7, G8).

At 28 days old, statistical analysis clarified significant difference at $(\mathrm{P}<0.05)$ among different groups in $\mathrm{X} \log$. vaccinated groups with live and double shot of inactivated vaccines continued to show the highest $\mathrm{Ab}$ titers among different groups as well as the levels of titers were increased compared to their level at age of 14 days reflecting higher protection level (X log $6.13 \pm 0.63 \mathrm{~A}$ and $6.13 \pm 0.62 \mathrm{~A}$ ) (G5, G6) compared to groups vaccinated with live vaccine only (X log $4.33 \pm 0.34 \mathrm{~B}$ and $4.26 \pm 0.33 \mathrm{~B})$ (G1, G2). Non vaccinated groups $(\mathbf{G 7}, \mathbf{G 8})$ are not only showed the lowest $\mathrm{Ab}$ levels but also the titers were decreased compared to those determined in 14 days old chicks (Table 1). 
On the other side, immune response to NDV vaccines at 35 days old (5 days post challenge) was tabulated in Fig. (1) Where statistical analysis clarified significant difference at $(\mathrm{P}<0.05)$ among different groups in $\mathrm{X} \log$. Vaccinated groups with live and double shot of inactivated vaccines continued to show the highest $\mathrm{Ab}$ titers among different groups but the levels of titers were decreased compared to their level at age of 28 days $(\mathrm{X}$ $\log 5.4 \pm 0.52 \mathrm{~A}$ and $5.53 \pm 0.53 \mathrm{~A})(\mathbf{G 5}, \mathbf{G 6})$ compared to groups vaccinated with live vaccine only (X $\log$ $3.53 \pm 0.55 \mathrm{C}$ and $3.26 \pm 0.32 \mathrm{C}$ ) (G1, G2). Moreover, it was recorded that chicks of control positive group (nonvaccinated and challenged by NDV) (G7) were totally dead. Administration of double shot of inactivated NDV vaccines (Inactivated GVII or Inactivated GII) and immunization with live NDV vaccine HB, ELITE and LaSota in 1,7, 10 and 18 days of life (G5, G6) induced high levels of NDV specific humoral antibodies and completely protect chicken from death post challenge with VV NDV challenge by the oculonasal route at $30^{\text {th }}$ day of age and reduce significantly the shedding of virus to the environment which subsequently decrease the No. of secondary infected birds.

Effects of different NDV vaccination programs on productive performance of broiler chicken during the experiment was recorded in Table (6), statistical analysis clarified significant difference among estimated FCR of all experimental groups. It was observed that FCR was better in groups vaccinated with live and inactivated vaccines (G3, G4, G5, G6) compared to those vaccinated with live vaccines only (G1, G2) (1.62 vs 1.7$)$ respectively indicated that combination of live and inactivated vaccines in immunization against NDV did not affect feed conversion ratio negatively.

Table 4: Mortality rate of chicken in different experimental groups after challenge (from day 1 post challenge to day 9 post challenge)

\begin{tabular}{|c|c|c|c|c|}
\hline \multirow{2}{*}{$\begin{array}{l}\text { Experimental Groups } \\
\text { (15 birds/group) }\end{array}$} & \multicolumn{2}{|c|}{ Dead birds } & \multicolumn{2}{|c|}{ Protection \% } \\
\hline & No. & $\%$ & No. & $\%$ \\
\hline Group 1 & 1 & 6.6 & 14 & 93.40 \\
\hline Group 2 & 2 & 13.3 & 13 & 86.60 \\
\hline Group 3 & 0 & 0.0 & 15 & 100.00 \\
\hline Group 4 & 0 & 0.0 & 15 & 100.00 \\
\hline Group 5 & 0 & 0.0 & 15 & 100.00 \\
\hline Group 6 & 0 & 0.0 & 15 & 100.00 \\
\hline Group 7 (control+ve) & 15 & 100.0 & 0 & 0.00 \\
\hline
\end{tabular}

Table 5: Oropharyngeal (tracheal) shedding of NDV from broiler chicken of different experimental groups at $3^{\text {rd }}, 5^{\text {th }}, 7^{\text {th }}$ and $9^{\text {th }}$ day

\begin{tabular}{|c|c|c|c|c|c|c|c|c|}
\hline G DPC & G 1 & G2 & G 3 & G 4 & G 5 & G 6 & G 7 & G 8 \\
\hline \multirow[t]{3}{*}{$3 \mathrm{DPC}$} & $15 / 15$ & $15 / 15$ & $10 / 15$ & $10 / 15$ & $10 / 15$ & $10 / 15$ & $15 / 15$ & $0 \%$ \\
\hline & $100 \%$ & $100 \%$ & $66.60 \%$ & $66.60 \%$ & $66.60 \%$ & $66.60 \%$ & $100 \%$ & \\
\hline & $3.29 \pm 1.03$ & $3.91 \pm 0.10$ & $2.92 \pm 0.81$ & $2.75 \pm 0.78$ & $1.67 \pm 0.76$ & $1.86 \pm 0.56$ & $4.27 \pm 0.15$ & \\
\hline \multirow[t]{3}{*}{$5 \mathrm{DPC}$} & $15 / 15$ & $15 / 15$ & $10 / 15$ & $10 / 15$ & $0 \%$ & $0 \%$ & $13 / 13$ & $0 \%$ \\
\hline & $100 \%$ & $100 \%$ & $66.60 \%$ & $66.60 \%$ & & & $100 \%$ & \\
\hline & $2.55 \pm 0.78$ & $2.92 \pm 1.21$ & $1.71 \pm 0.83$ & $1.65 \pm 0.83$ & & & $4.27 \pm 0.15$ & \\
\hline \multirow[t]{3}{*}{ 7DPC } & $4 / 14$ & $10 / 13$ & $5 / 15$ & $5 / 15$ & $0 \%$ & $0 \%$ & $0 \%$ & $0 \%$ \\
\hline & $28.50 \%$ & $77 \%$ & $33.30 \%$ & $33.30 \%$ & & & & \\
\hline & $1.68 \pm 0.53$ & $1.86 \pm 0.56$ & $1.67 \pm 0.31$ & $1.05 \pm 0.43$ & & & & \\
\hline \multirow[t]{3}{*}{ 9DPC } & $3 / 14$ & $4 / 13$ & $0 \%$ & $0 \%$ & $0 \%$ & $0 \%$ & $0 \%$ & $0 \%$ \\
\hline & $21.40 \%$ & $30.77 \%$ & & & & & & \\
\hline & $1.05 \pm 0.41$ & $1.25 \pm 0.83$ & & & & & & \\
\hline
\end{tabular}

*The mean shedding titer =SUM of virus shedding/ total Number of shedders. Virus shedding titer (titer \pm SD) as EID 50 using qRTPCR with absolute stander curve and the engine detection threshold 0.7 EID 50

Table 6: Effects of different NDV vaccination programs on productive performance of broiler chicken in different experimental groups

\begin{tabular}{llll}
\hline Experimental Groups & $\begin{array}{l}\text { Final Body weight } \\
(\mathrm{g}) \text { at 39 days old }\end{array}$ & Total Feed consumption $(\mathrm{g})$ & Feed conversion rate $($ FCR) \\
\hline Group 1 & $2120 \pm 21.20 \mathrm{C}$ & $3610 \pm 34.35 \mathrm{D}$ & $1.7 \pm 0.18 \mathrm{~A}$ \\
Group 2 & $2095 \pm 25.55 \mathrm{D}$ & $3600 \pm 33.35 \mathrm{D}$ & $1.71 \pm 0.17 \mathrm{~A}$ \\
Group 3 & $2220 \pm 22.24 \mathrm{~B}$ & $3650 \pm 35.60 \mathrm{C}$ & $1.64 \pm 0.16 \mathrm{~B}$ \\
Group 4 & $2235 \pm 23.24 \mathrm{~B}$ & $3660 \pm 36.60 \mathrm{C}$ & $1.63 \pm 0.18 \mathrm{~B}$ \\
Group 5 & $2275 \pm 27.25 \mathrm{~B}$ & $3700 \pm 33.37 \mathrm{~B}$ & $1.62 \pm .16 \mathrm{~B}$ \\
Group 6 & $2265 \pm 26.27 \mathrm{~B}$ & $3690 \pm 33.38 \mathrm{~B}$ & $1.62 \pm .16 \mathrm{~B}$ \\
Group 7 (control + ve) & All chicken dead & & \\
Group 8 (control - ve) & $2440 \pm 22.34 \mathrm{~A}$ & $3760 \pm 33.63 \mathrm{~A}$ & $1.54 \pm 0.17 \mathrm{~B}$ \\
\hline
\end{tabular}




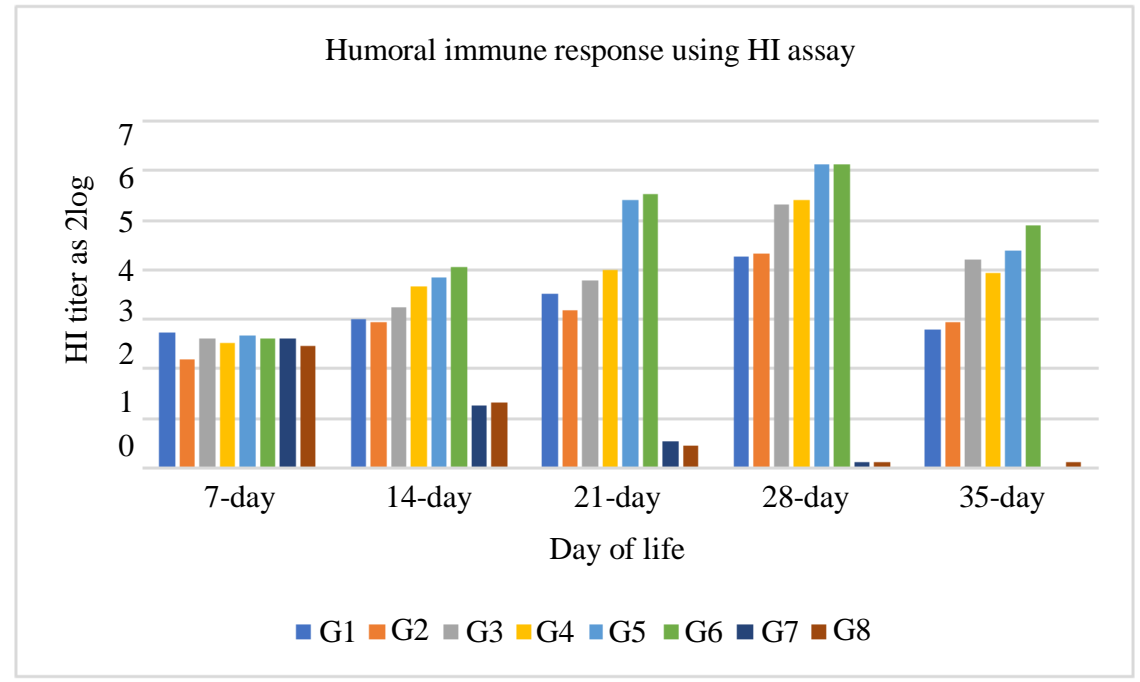

Fig. 1: Humoral Immune response for ND using HI assay along the experiment period Discussion

\section{Discusion}

Newcastle Newcastle disease (ND) is one of the most important contagious diseases of poultry and other bird species and considered as a global threat to commercial poultry production (Alexander and Senne, 2008). Velogenic strains of ND virus cause a devastating disease of poultry. Genotype VII (class II genotype VII) was firstly categorized into two sub genotypes: VIIa, which represents viruses that emerged in the 1990s in the Far East and spread to Europe and Asia; and VIIb, which represents viruses that emerged in the Far East and spread to South Africa. Later, the sub genotypes of VII are classified into VIIc, d, and e, which represent isolates from China, Kazakhstan and South Africa and VIIf, g, h and i, which represent African isolates (de Almeida et al., 2009; Miller et al., 2009). The phylogenetic analysis showed Egyptian NDV isolates are closely related with the genotype II of class II NDV strains. So, sequences of the F genes of 2006 Egypt isolates are closely related to that of the 2005 suggesting that these strains are probably circulating in the vaccinated bird population in Egypt until development of an outbreak (Mohamed et al., 2011). Different live and inactivated NDV vaccines were used throughout the experiment including; NDHB1, ELITE, LaSota, Inactivated GII and Inactivated GVII. Broiler chicks were divided into 8 groups (150 chicks / group); 6 groups undergo different vaccination programs against NDV while 2 groups were kept without vaccination to be the control groups. Challenge was done at day 30 to groups from 1 to 7 via intranasal administration of NDV $\left(10^{6} \mathrm{EID}_{50}\right)$ velogenic GVII (NDV/CK/Egypt/567F/2012). Firstly, it was found that broiler chicken of the control negative group that was neither vaccinated nor challenged were all alive throughout the experiment (G8). There are two methods for detection of ND Virus shedding, NDV titration in viral samples in embryonated chicken eggs (ECE) or in tissue culture, which is time consuming and costly. Recently, a Quantitative real-time RT-PCR (RRT-PCR) based assay has been developed and validated for detection of NDV shedding (Perozo et al., 2012). The amount of NDV shed into the environment by vaccinated birds has arisen as a potential indicator of vaccine efficacy (Miller et al., 2009; Miller et al., 2007). Using vaccines formulated with a NDV with the same (homologous) genotype of NDV challenge virus, for both genotype II and genotype V NDV isolates, is possible to decrease not only the number of birds shedding NDV, but also the amount of NDV shed from individual birds by evaluating oropharyngeal swab material, However, in those studies, the amount of virus shed from the birds vaccinated with vaccines heterologous to the genotype of the challenge virus was also decreased but at lower amounts (Miller et al., 2007). Like most vaccines, NDV vaccines do not prevent vaccinated birds from becoming infected with NDV and subsequent shedding of the virus (Kapczynski and King, 2005). However, most vaccines will significantly decrease the amount of virus shed in saliva and feces compared to unvaccinated birds (Miller et al., 2009). The amount shed will depend on the immunity of the host, the host species infected, the amount and virulence of the challenge virus, the dose and type of ND vaccine and the time between vaccination and challenge, While the amount will vary depending on the NDV isolate and the host species (Miller et al., 2013b). The recorded results in Table (5) showed the Oropharyngeal (tracheal) shedding of challenged NDV from broiler chicken at $3^{\text {rd }}$ day post challenge. $\mathrm{Chi}^{2}$ analysis of the obtained results showed significant difference between the shedding titers of NDV of different experimental groups where the 
lowest shedding titer was recorded in experimental group that was vaccinated by $\mathrm{HB}+$ Inactivated GVII, Elite, Inactivated GVII and LaSota vaccines at days 1, 7, 10 and 18 of age (G5), respectively followed by the experimental group vaccinated by $\mathrm{HB}+$ Inactivated GII, Elite, Inactivated GII and LaSota at the same age (G6), respectively. On the other hand, the highest shedding titer was recorded in experimental group that was not vaccinated and challenged by NDV (G7) followed by the experimental group that was vaccinated by $\mathrm{HB}$ and LaSota vaccines at days 1 and 18 of age (G2), respectively. As shown in Table (5), it was observed that experimental groups that was vaccinated by $\mathrm{HB}+$ Inactivated GVII, Elite, Inactivated GVII and LaSota vaccines (G5) and $\mathrm{HB}$ + Inactivated GII, Elite, Inactivated GII and LaSota (G6) at days 1, 7, 10 and 18 of age, respectively showed no shedding of the NDV at $5^{\text {th }}$ DPC reflecting efficient vaccination program. On the other hand, the highest shedding titer $(4.27 \pm 0.15 \mathrm{~A})$ was recorded in experimental group that was not vaccinated and challenged by NDV (G7) followed by the experimental group that was vaccinated by $\mathrm{HB}$ and LaSota vaccines $(2.92 \pm 1.21 \mathrm{~B})$ at days 1 and 18 of age (G2), respectively. Moreover, it was recorded that birds of the group that was not vaccinated and challenged by NDV were all dead as a result of ND infection (G7). The presented data in Table (5) clarified the Oropharyngeal (tracheal) shedding of challenged NDV from broiler chicken at $7^{\text {th }}$ DPC. As $5^{\text {th }}$ DPC, it was observed that experimental groups that was vaccinated by $\mathrm{HB}+$ Inactivated GVII, Elite, Inactivated GVII and LaSota vaccines (G5) and $\mathrm{HB}+$ Inactivated GII, Elite, Inactivated GII and LaSota (G6) at days 1, 7, 10 and 18 of age, respectively showed no shedding of the NDV reflecting efficient vaccination program. On the other hand, the highest shedding titer $(1.86 \pm 0.56 \mathrm{~A})$ was recorded in experimental group that was vaccinated by $\mathrm{HB}$ and LaSota vaccines at days 1 and 18 of age (G2), respectively followed by experimental group that was vaccinated by $\mathrm{HB}$, Elite and LaSota (G1) $(1.68 \pm 0.53 \mathrm{~A})$. At $9^{\text {th }}$ DPC, viral shedding occurred only in the group vaccinated by $\mathrm{HB}$ and LaSota at days of 1 and 18 of age (G2) by shedding birds $(30.7 \%)$ and shedding titer of $(1.25 \pm 0.83 \mathrm{~A})$ and the group vaccinated by $\mathrm{HB}$, Elite and LaSota at days of 1,7 and 18 of age (G1) by only shedding birds $(21.4 \%)$ and shedding titer of $(1.05 \pm 0.41 \mathrm{~A})$ (Table 5), which agreed with Similar findings were in previous reports (Cho et al., 2007; Jeon et al., 2008; Kapczynski and King, 2005; Van Boven et al., 2008; Yu et al., 2001). Collectively, the results indicated that the used vaccination program provides chicken with protection from disease caused by the genotype VII virus, as no mortality or disease symptoms were observed in any of the vaccinated chicken. The results of this study therefore contrast with the concerns in the field and published reports that, current ND vaccines may not produce adequate protection against velogenic challenge (Liu et al., 2003). Mortality rate of broiler chicken during the experiment was tabulated in Tables (4). Concerning mortality rate of broiler chicken after challenge by NDV, the highest mortality rate $(100 \%)$ was observed in non-vaccinated challenged group (G7) where no vaccination programs were applied. On contrary, no mortalities (100\% protection rate) were recorded in groups vaccinated with both live and inactivated NDV vaccines (G3, G4, G5, and G6). In-addition, low mortality rates recorded in groups vaccinated with live vaccines only $(\mathbf{G 1}, \mathbf{G 2})$ by (HB, Elite and LaSota). Yi et al., (2011) proved that LaSota and ELITE strain of NDV vaccines not effective after VIId NDV strain isolated from chicken in Shanghai, China which has caused several breaks in China and Taiwan (Yi et al., 2011). Blood samples were taken from chicken groups before and after challenge, antibodies to NDV were quantified by hemagglutination inhibition test (HI), serum samples were collected and prepared 5-day post challenge from vaccinated challenged and nonchallenged chicks. Protection from ND correlates with $\mathrm{HI}$ antibody titers at the day of challenge (Kapczynski and King, 2005; Kapczynski et al., 2006), All birds in the vaccinated groups developed protective titers from humoral immune response measured by $\mathrm{HI}$ assay in comparison to non-vaccinated group as previously described " over $4 \log _{2}$ " (Saidu et al., 2003). The immune response to NDV vaccines was determined using HI test. Approximately, $5 \%$ (60/1200) of 1-day old randomly selected chicks were tested by HI test for determination of maternal immunity and it was found that X Log titer was 5.8. The presented data in Fig. (1) Showed that 15 randomly selected chicks from each experimental group (150 chicks) at 14 days old were tested by $\mathrm{HI}$ test for determination of $\mathrm{Ab}$ titers against VDV vaccine. Statistical analysis clarified significant difference at $(\mathrm{P}<0.05)$ among different groups in $\mathrm{X} \log$. The highest $\mathrm{Ab}$ titers was observed in group vaccinated with $\mathrm{HB}+$ Inactivated GII, Elite, Inactivated GII and LaSota vaccines (G6) (Live and double inactivated GII vaccine) (X $\log 4.06 \pm 0.44 \mathrm{~A})$ followed by the group vaccinated with $\mathrm{HB}+$ Inactivated GVII, Elite, Inactivated GVII and LaSota (G5) (Live and double shot of inactivated GVII vaccine) (X $\log 3.86 \pm 0.85 \mathrm{~B})$. On contrary, positive and negative control groups (nonvaccinated) showed the lowest $\mathrm{Ab}$ titers (X Log $1.26 \pm 0.20 \mathrm{~F}$ and $1.33 \pm 0.20 \mathrm{~F}$, respectively) (G7, G8). At 28 days old, statistical analysis clarified significant difference at $(\mathrm{P}<0.05)$ among different groups in $\mathrm{X} \log$. vaccinated groups with live and double shot of inactivated vaccines continued to show the highest $\mathrm{Ab}$ titers among different groups as well as the levels of titers were increased compared to their level at age of 14 days reflecting higher protection level (X log $6.13 \pm 0.63 \mathrm{~A}$ and $6.13 \pm 0.62 \mathrm{~A})(\mathbf{G 5}$, G6) compared to groups vaccinated with live vaccine only (X log 4.33 $\pm 0.34 \mathrm{~B}$ and $4.26 \pm 0.33 \mathrm{~B})(\mathbf{G 1}, \mathbf{G 2})$. Non vaccinated groups $(\mathbf{G 7}, \mathbf{G 8})$ are not only showed the lowest $\mathrm{Ab}$ levels but also the titers were decreased compared to 
those determined in 14 days old chicks Fig. (1). On the other side, immune response to NDV vaccines at 35 days old (5 days post challenge) was tabulated in Fig. (1) Where statistical analysis clarified significant difference at $(\mathrm{P}<0.05)$ among different groups in $\mathrm{X} \log$. Vaccinated groups with live and double shot of inactivated vaccines continued to show the highest $\mathrm{Ab}$ titers among different groups but the levels of titers were decreased compared to their level at age of 28 days ( $\mathrm{X}$ $\log 5.4 \pm 0.52 \mathrm{~A}$ and $5.53 \pm 0.53 \mathrm{~A})$ (G5, G6) compared to groups vaccinated with live vaccine only (X log $3.53 \pm 0.55 \mathrm{C}$ and $3.26 \pm 0.32 \mathrm{C}$ ) (G1, G2). Moreover, it was recorded that chicks of control positive group (nonvaccinated and challenged by NDV) (G7) were totally dead. Administration of double shot of inactivated NDV vaccines (Inactivated GVII or Inactivated GII) and immunization with live NDV vaccine $\mathrm{HB}$, Elite and LaSota in 1,7, 10 and 18 days of life (G5, G6) induced high levels of NDV specific humoral antibodies and completely protect chicken from death post challenge with VV NDV challenge by the oculonasal route at $30^{\text {th }}$ day of age and reduce significantly the shedding of virus to the environment which subsequently decrease the No. of secondary infected birds. The level of protection achieved in this study demonstrates the efficacy of the test vaccine program based on combination between live and inactivated vaccines (either GVII or GII) and its ability to protect against the clinical disease of ND provide significant higher protection level in comparison to using live vaccine alone. This immunization procedure can be recommended for prevention of GVII NDV strain and this finding agreed with previous reports (Bennejean et al., 1978); who proved that simultaneous vaccination of day-old chicks with live and inactivated ND vaccines results in better protection as compared with a single vaccination. Poor vaccine immune response is responsible for viral shedding rather than variation in virus antigenicity and the proper vaccine program can reduce shedding and in turn decrease the environmental virus load and decrease the chance to develop reservoirs. Effects of different NDV vaccination programs on productive performance of broiler chicken during the experiment was recorded in Table (6), statistical analysis clarified significant difference among estimated FCR of all experimental groups. It was observed that FCR was better in groups vaccinated with live and inactivated vaccines (G3, G4, G5, G6) compared to those vaccinated with live vaccines only (G1, G2) (1.62 vs 1.71) respectively indicated that combination of live and inactivated vaccines in immunization against NDV did not affect feed conversion ratio negatively.

\section{Conclusion}

The the combination between live and inactivated ND vaccine provide significantly better protection in broiler chicken in comparison of using live vaccine alone and there is no significant difference between the different genotypes of inactivated ND vaccine as both inactivated genotype-II and Genotype-VII within the vaccination program of ND virus have similar protective levels in broiler chicks.

\section{Author's Contributions}

Mousa A. Ayoub, Wael Elfeil, Diaa El Boraey: Conceived and designed the experiments.

Mousa A. Ayoub, Wael Elfeil, Diaa El Boraey: performed the experiments.

Mousa A. Ayoub, Wael Elfeil, Diaa El Boraey Haitham Hammam and Mohamed, A. Nossair: Analyzed the data.

Mousa A. Ayoub, Wael Elfeil, Diaa El Boraey: Contributed reagents/materials/analysis tools.

Wael Elfeil, Haitham Hammam and Mohamed, A. Nossair: Wrote and review the manuscript.

\section{Conflict of Interest}

The authors declare that there is no conflict of interests regarding the publication of this paper.

\section{References}

Abouelmaatti, Reham R, Abdelazeem M Algammal, Xiaokun Li, Jisheng Ma, Eman A Abdelnaby, and Wael MK Elfeil. 2013. 'Cloning and analysis of Nile tilapia Toll-like receptors type-3 mRNA. Central European Journal of Immunology, 38: 277-82.

Alexander, D. and D. Senne, 2008. Newcastle Disease and Other Paramyxoviruses. In: A Laboratory Manual for the Isolation Identification and Characterization of Avian Pathogens, Swayne, D.E., J.E. Pearson, W.M. Reed, W.M. Jackwood and P.R. Woolcock et al. (Eds.), Omni Press, Inc, pp: 135-41.

Bennejean, G., M. Guittet, J. Picault, J. Bouquet and B. Devaux et al., 1978. Vccination of one-day-old chicks against newcastle disease using inactivated oil adjuvant vaccine and/or live vaccine. Avian Pathol., 7: 15-27. DOI: 10.1080/03079457808418256

Cho, S.H., S.J. Kim and H.J. Kwon, 2007. Genomic sequence of an antigenic variant Newcastle disease virus isolated in Korea. Virus Genes, 35: 293-302.

Daubney, R. and W. Mansy, 1948. The occurrence of Newcastle disease in Egypt. J. Comparative Pathol. Therapeutics, 58: 189-200.

De Almeida, R.S., O.F. Maminiaina, P. Gil, S. Hammoumi and S. Molia et al., 2009. Africa, a reservoir of new virulent strains of Newcastle disease virus? Vaccine, 27: 3127-3129.

Desingu, P., S. Singh, K. Dhama, O.V. Kumar and Y. Malik and R. Singh, 2017. Clinicopathological characterization of experimental infection in chickens with sub-genotype VIIi Newcastle disease virus isolated from peafowl. Microbial. Pathogenesis, 105: 8-12. 
Diab, M.S., M.S. Abd-ElHafez, M.A. Ashry and W.K. Elfeil, 2019. Occurrence of avian influenza H5N1 among chicken, duck farms and human in Egypt. Am. J. Anim. Veterinary Sci.

Dimitrov, K.M., C.L. Afonso, Q. Yu and P.J. Miller, 2017. Newcastle disease vaccines-a solved problem or a continuous challenge? Vet. Microbiol., 206: 126-136.

Eid, H.I., A.M. Algammal, S.A. Nasef, W.K. Elfeil and G.H. Mansour, 2016. Genetic variation among avian pathogenic E. coli strains isolated from broiler chickens. Asian J. Anim. Vet. Adv., 11: 350-356.

Eid, H.M., A.M. Algammal, W.K. Elfeil, F.M. Youssef and S.M. Harb et al., 2019. Prevalence, molecular typing and antimicrobial resistance of bacterial pathogens isolated from ducks. Vet.World, 12: 677-683.

Elfeil, W. K., R. R. Abouelmaatti, C. Sun, W. Han, X. Li, J. Ma, L. Lei, S. Liu, Y. Yang, Y. Wang, M. Mandour, M. Fawzy, and M. N. Shalaby. 2012. 'Identification, cloning, expression of a novel functional anasplatyrhynchos mRNA TLR4. Journal of Animal and Veterinary Advances, 11: 1727-33.

Elfeil, Wael M.K., Abdelazeem M. Algammal, Reham R. Abouelmaatti, Ahmed Gerdouh, and Mohamed Abdeldaim. 2016. 'Molecular characterization and analysis of TLR-1 in rabbit tissues. Central European Journal of Immunology, 41: 236-42.

Enany, Mohamed E., Abdelazeem M. Algammal, Amr M. Hanora, Gehan I. Shagar, Wael K. Elfeil, and Noha M. Elshaffy. 2018. 'Molecular Typing And Evaluation Of Sidr Honey Inhibitory Effect On Virulence Genes Of MRSA Strains Isolated From Catfish In Egypt. Pak. J. Pharmaceutical Sci., 31: 1865-70.

Elhady, M.A., A. Ali, W.H. Kilany, W.K. Elfeil and H. Ibrahim et al., 2018. Field efficacy of an attenuated infectious bronchitis variant 2 virus vaccine in commercial broiler chickens. Vet. Sci., 5: 49.

Guo, H, X. Liu, Y. Xu, Z. Han and Y. Shao et al., 2014. A comparative study of pigeons and chickens experimentally infected with PPMV-1 to determine antigenic relationships between PPMV-1 and NDV strains. Vet. Microbiol., 168: 88-97.

Institute, S., 2014. SAS 9.4 Output delivery system: User's guide. SAS Institute.

Jeon, W.J., E.K. Lee, Y.J. Lee, O.M. Jeong and Y.J. Kim et al., 2008. Protective efficacy of commercial inactivated Newcastle disease virus vaccines in chickens against a recent Korean epizootic strain. J. Vet. Sci., 9: 295-300.

Kapczynski, D.R. and D.J. King, 2005. Protection of chickens against overt clinical disease and determination of viral shedding following vaccination with commercially available Newcastle disease virus vaccines upon challenge with highly virulent virus from the California 2002 exotic Newcastle disease outbreak. Vaccine, 23: 3424-3433.
Kapczynski, D.R., M.G. Wise and D.J. King, 2006. Susceptibility and protection of naive and vaccinated racing pigeons (Columbia livia) against exotic Newcastle disease virus from the California 2002-2003 outbreak. Avian Dis., 50: 336-341.

Kärber, G., 1931. Beitrag zur kollektiven Behandlung pharmakologischer Reihenversuche. Arch Exp. Pathol. Pharmakol., 162: 480-483.

Liu, M., Y. Qu, F. Wang, S. Liu and H. Sun, 2015. Genotypic and pathotypic characterization of Newcastle disease virus isolated from racing pigeons in China. Poult. Sci., 94: 1476-1482.

Liu, X, H. Wan, X. Ni, Y Wu and W. Liu, 2003. Pathotypical and genotypical characterization of strains of Newcastle disease virus isolated from outbreaks in chicken and goose flocks in some regions of China during 1985-2001. Arch Virol., 148: $1387-1403$.

Mansur-Ud-Din, A., M. Chaudhry, M.F. Rai and H.B. Rashid, 2007. Evaluation of two vaccination schemes using live vaccines against Newcastle disease in chickens. Turkish J. Vet. Anim. Sci., 31: 165-169.

Marangon, S. and L. Busani, 2007. The use of vaccination in poultry production. Revue Sci. Technique-Office Int. Epizooties, 26: 265-265.

Miller, P.J., C.L. Afonso, J. El Attrache, K.M. Dorsey and S.C. Courtney et al., 2013a. Effects of Newcastle disease virus vaccine antibodies on the shedding and transmission of challenge viruses. Dev. Comp. Immunol., 41: 505-13.

Miller, P.J., C. Estevez, Q. Yu, D.L. Suarez and D.J. King, 2009. Comparison of viral shedding following vaccination with inactivated and live Newcastle disease vaccines formulated with wild-type and recombinant viruses. Avian Dis., 53: 39-49.

Miller, P., D.J. King, C.L. Afonso and D.L. Suarez, 2007. Antigenic differences among Newcastle disease virus strains of different genotypes used in vaccine formulation affect viral shedding after a virulent challenge. Vaccine, 25: 7238-7246.

Mohamed, M.H., S. Kumar, A. Paldurai and S.K. Samal, 2011. Sequence analysis of fusion protein gene of Newcastle disease virus isolated from outbreaks in Egypt during 2006. Virol. J., 8: 237-237.

Nwanta, J., P. Abdu and W. Ezema, 2008. Epidemiology, challenges and prospects for control of Newcastle disease in village poultry in Nigeria. World's Poultry Sci. J., 64: 119-127.

OIE, 2015. Manual of Diagnostic Tests and Vaccines for Terrestrial Animals-Avian Influenza: OIE Terrestrial Manual.

Orsi, M., J. Doretto, L. Reischak, D. da Silva and L, Spilki et al., 2009. Newcastle disease virus vaccine strains: Immunogenicity is not influenced by ICPI. Brazilian J. Poultry Sci., 11: 129-133. 
Perozo, F., R. Marcano and C.L. Afonso, 2012 Biological and phylogenetic characterization of a genotype VII Newcastle disease virus from Venezuela: Efficacy of field vaccination. J. Clin. Microbiol., 50: 1204-1208.

Sabry, M., 2019. Current situaton of poultry industry in Egypt. In: EVPA, Salaheldein, H. (Ed.), Porto Marina, Egypt.

Saidu, L., A. Wakawa and P. Abdu, 2003. Snake bite in a multi-specie backyard poultry in Zaria: A case report. Sokoto J. Vet. Sci., 5: 19-21.

Sedeik, M.E., A.M. Awad, H. Rashed and W.K. Elfeil, 2018. Variations in pathogenicity and molecular characterization of Infectious Bursal Disease Virus (IBDV) in Egypt. Am. J. Anim. Vet. Sci., 13: 76-86.

Sedeik, M.E., A. Nahed, A.M. Awad, H.S. AbdElhamid and H.F. Ellakany et al., 2019. Identifying intra-specific variability in the virulence of eimeria tenella using SCAR markers. Poult. Sci., 18: 151-158.

Senne, D., D. King and D. Kapczynskio, 2004. By Vaccination. Dev. Biol. Basel., 119: 165-170.
Sultan, H.A.E., A.M. Arafa, M. Talaat, A.A.E.R. Gaballa and W.H. Kilany et al., 2019. Efficacy of Clade2. 3.2 H5-recombinant baculovirus vaccine in protecting muscovy and pekin ducks from clade 2.3. 4.4 H5N8 highly pathogenic avian influenza infection. Avian Dis., 61

Swayne, D.E. and J.R. Glisson, 2013. Diseases of Poultry. 1st Edn., Wiley-Blackwell, Ames, Iowa.

Van Boven, M., A. Bouma, T.H. Fabri, E. Katsma and L. Hartog et al., 2008. Herd immunity to Newcastle disease virus in poultry by vaccination. Avian Pathol., 37: 1-5.

Yi, J., C. Liu, B. Chen and S. Wu, 2011. Molecular characterization of a virulent genotype VIId strain of Newcastle disease virus from farmed chickens in Shanghai. Avian Dis., 55: 279-284.

Yu, L, Z. Wang, Y. Jiang, L. Chang and J. Kwang, 2001. Characterization of newly emerging Newcastle disease virus isolates from the people's republic of China and Taiwan. J. Clin. Microbiol., 39: 3512-3519. 